\title{
Anterior Cranial Fossa Calcifying Pseudoneoplasm of the Neuroaxis-Diagnosis Using a Transblepharoplasty Approach
}

\author{
Timothy C. Blood ${ }^{1} \quad$ Fausto J. Rodriguez ${ }^{2}$ Norris Nolan ${ }^{3}$ Murugappan Ramanathan Jr. ${ }^{4}$ Shaun C. Desai ${ }^{5}$
}

${ }^{1}$ Department of Otolaryngology-Head and Neck Surgery, Walter Reed National Military Medical Center, Bethesda, Maryland, United States

2 Department of Pathology, Johns Hopkins University School of Medicine, Johns Hopkins University, Baltimore, Maryland, United States

${ }^{3}$ Department of Pathology, Johns Hopkins University School of Medicine, Suburban Hospital, Bethesda, Maryland, United States

${ }^{4}$ Department of Neurosurgery, Johns Hopkins University School of Medicine, Johns Hopkins University, Baltimore, Maryland, United States

${ }^{5}$ Department of Otolaryngology-Head and Neck Surgery, Johns Hopkins University School of Medicine, Johns Hopkins University, Baltimore, Maryland, United States

J Neurol Surg Rep 2018;79:e75-e78.
Address for correspondence Timothy C. Blood, MD, Department of Otolaryngology-Head and Neck Surgery, Walter Reed National Military Medical Center, 8901 Rockville Pike, Bethesda, MD, United States (e-mail: timothyblood333@gmail.com).
Abstract
Keywords
- CAPNON
- calcifying pseudoneoplasms of the neuroaxis
- transblepharoplasty
- frontal sinus
- anterior skull base
- minimally invasive

Calcifying pseudoneoplasms of the neuroaxis (CAPNON) are rare, benign tumors of unknown histogenesis. CAPNON generally are found intracranially or within the spinal column in symptomatic patients. We present the case of an asymptomatic patient with an incidentally discovered right anterior cranial fossa mass with extension through the posterior and anterior table of the right frontal sinus and right superior orbital roof. Open biopsy was performed via a transblepharoplasty incision with pathological diagnosis of CAPNON. The biopsy approach was well hidden and resulted in minimal to no postoperative scarring and little postoperative pain. We present the first documented case of CAPNON involving the frontal sinus via the anterior cranial base. Given our experience, in a patient with a mass involving the frontal sinus and superior orbital rim, the transblepharoplasty approach provides excellent exposure and access for pathological diagnosis. Further, we recommend that CAPNON remain on the differential for aggressive appearing calcified masses of the anterior cranial fossa.

\section{Introduction}

Calcifying pseudoneoplasms of the neuroaxis (CAPNON) are rare, benign lesions with no known neoplastic capability. First described in 1978, CAPNON has been reported 59 times in the literature. ${ }^{1,2}$ The tissue of origin is postulated to be arachnoid mater or fibroblasts in the choroid plexus stroma. ${ }^{2}$ While the underlying etiology for the development of CAPNON is not fully understood, it is thought that they occur as response to trauma, infection or inflammation, or even tumors. $^{3}$ They occur with equal incidence in the spinal column and intracranially and have been documented to be found in patients from age 6 to $90 .^{1,4}$ To date, there has only been one case reported of a CAPNON of the anterior skull base (ethmoid cavity) and no reported cases involving the frontal sinus. ${ }^{5}$ Most of the CAPNONs in the literature were found secondary to locally compressive symptoms (pain, radiculopathy, or seizures); however, García Duque et al did note 3 cases which were discovered incidentally on imaging. ${ }^{1}$ Historically, the treatment of CAPNON has been total resection with subtotal resection allowable to preserve vital structures. Generally, excision at the time of diagnosis is received

February 13, 2018

accepted

June 29, 2018
DOI https://doi.org/

10.1055/s-0038-1673392. ISSN 2193-6358. (c) 2018 Georg Thieme Verlag KG
Stuttgart · New York

License terms

(1) $\Theta \circledast$ 
undertaken to relieve compressive symptoms. However, in a patient without symptoms, measured biopsy and planning secondary to pathological analysis is reasonable.

Approaches to the superior orbital rim are numerous and include: endoscopic, coronal, and direct brow. In 2007, the transblepharoplasty approach was reported as a cosmetically favorable approach to clearing pathology in the lateral frontal sinus. ${ }^{6}$ This approach was further refined as an aesthetically appealing approach to anterior skull base lesions. $^{7-10}$ The authors remarked on the favorable postoperative healing and advantageous cosmetic result. We used this approach to obtain a pathological diagnosis thereby obviating the need for a more invasive surgery or subjecting the patient to a cosmetically unacceptable outcome given the benign pathology.

\section{Case Report}

A 65-year-old female presented to an outside clinic with complaints of hearing loss. As part of the workup, a computed tomography (CT) of the head with and without contrast was obtained with incidental finding of an illdefined area of decreased attenuation of the right frontal and subfrontal regions. A follow-up magnetic resonance imaging of the brain with and without contrast was next obtained which showed a right frontal lobe vasogenic pattern abnormality with adjacent, well-circumscribed, irregular calcific, and/or remote hemorrhagic mass measuring $1.2 \mathrm{~cm} \times 1.6 \mathrm{~cm} \times 1.2 \mathrm{~cm}$ abutting the dura and eroding the posterior table of the frontal sinus. At this time she was referred to our clinic for further evaluation and management of the mass, which was thought to be malignant.

On presentation to our clinic, she was asymptomatic. To better characterize the mass and the surrounding bony anatomy for surgical planning, a fine cut sinus noncontrast CT was obtained showing a $1.3 \mathrm{~cm} \times 1.0 \mathrm{~cm}$ ossified mass seemingly centered in the right frontal sinus, at the level of the brow, with clear erosion of the anterior and posterior tables and erosion of the superior orbital roof (-Fig. 1). Given the radiologic findings, a biopsy was planned for pathological diagnosis and planning purposes.
Given the position of the mass within the frontal sinus and at the superior orbital rim, a transblepharoplasty approach was chosen to access the mass.

After infiltrating local anesthetic into the supratarsal crease, a skin incision was performed and carried through the orbicularis oculi with careful attention paid to not disrupt the orbital septum. The skin-muscle flap was bluntly raised to identify the superior orbital rim. Electrocautery was used to incise the periosteum of the superior orbital rim and a subperiosteal dissection was performed. The supratrochlear neurovascular bundle was specifically identified ( $\mathbf{F i g .} \mathbf{2 B}$ ), and branches of the artery and vein were cauterized with careful attention paid to preserve the nerve. Using Fusion CT-guided navigation (Medtronic, Dublin, Ireland) for confirmation, the mass was identified. The mass was biopsied $(\sim 1 \mathrm{~cm} \times 1 \mathrm{~cm})$ and sent for permanent pathology. No cerebrospinal fluid was noted after obtaining the biopsy. Hemostasis was achieved with cautery. The muscle and skin were then closed in layers (-Fig. $2 \mathbf{C}$ ).

Pathology revealed central calcification with surrounding chondroid matrix organized in a nodular pattern. In addition, bland spindle cell component with palisading around the nodules was noted (-Fig. 3). An epithelial membrane antigen (EMA) immunohistochemical stain was positive (not shown). These radiological and histological findings were consistent with the diagnosis of a CAPNON. After a long discussion with the patient, she elected to undergo conservative management with close interval repeat CT scans to assess for interval growth. After several months of follow-up, the patient has healed well with no complications, and a cosmetically acceptable result.

\section{Discussion}

The vast majority of CAPNON are discovered due to locally compressive symptoms, while the discovery of CAPNON as an incidental finding is rare. ${ }^{1}$ On imaging, the mass was aggressive appearing which prompted an expeditious evaluation to obtain a pathological diagnosis. Consistent with existing reports of CAPNON, the mass was locally erosive with obvious calcifications and appeared to be congruent with the meninges. A transblepharoplasty approach to the superior orbital rim and the anterior table of the frontal sinus gave
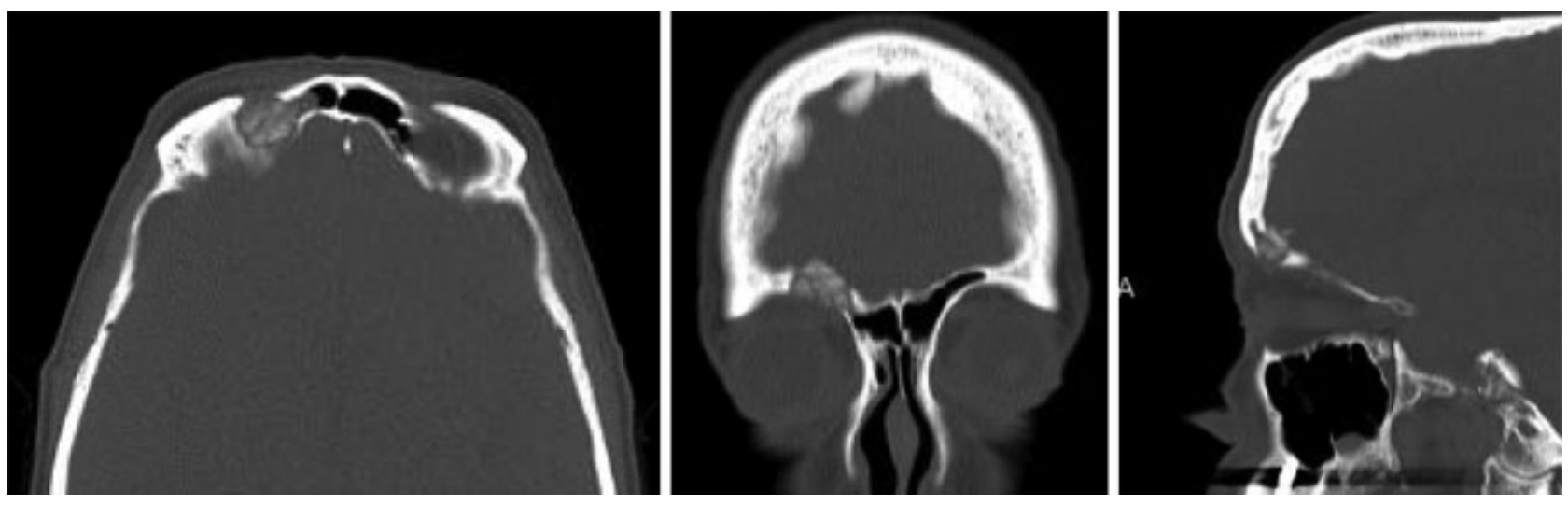

Fig. 1 Computed tomography (CT) sinus fine cut protocol showing ossified mass seemingly centered in the right frontal sinus, at the level of the brow, with clear erosion of the anterior and posterior tables and erosion of the superior orbital roof. 

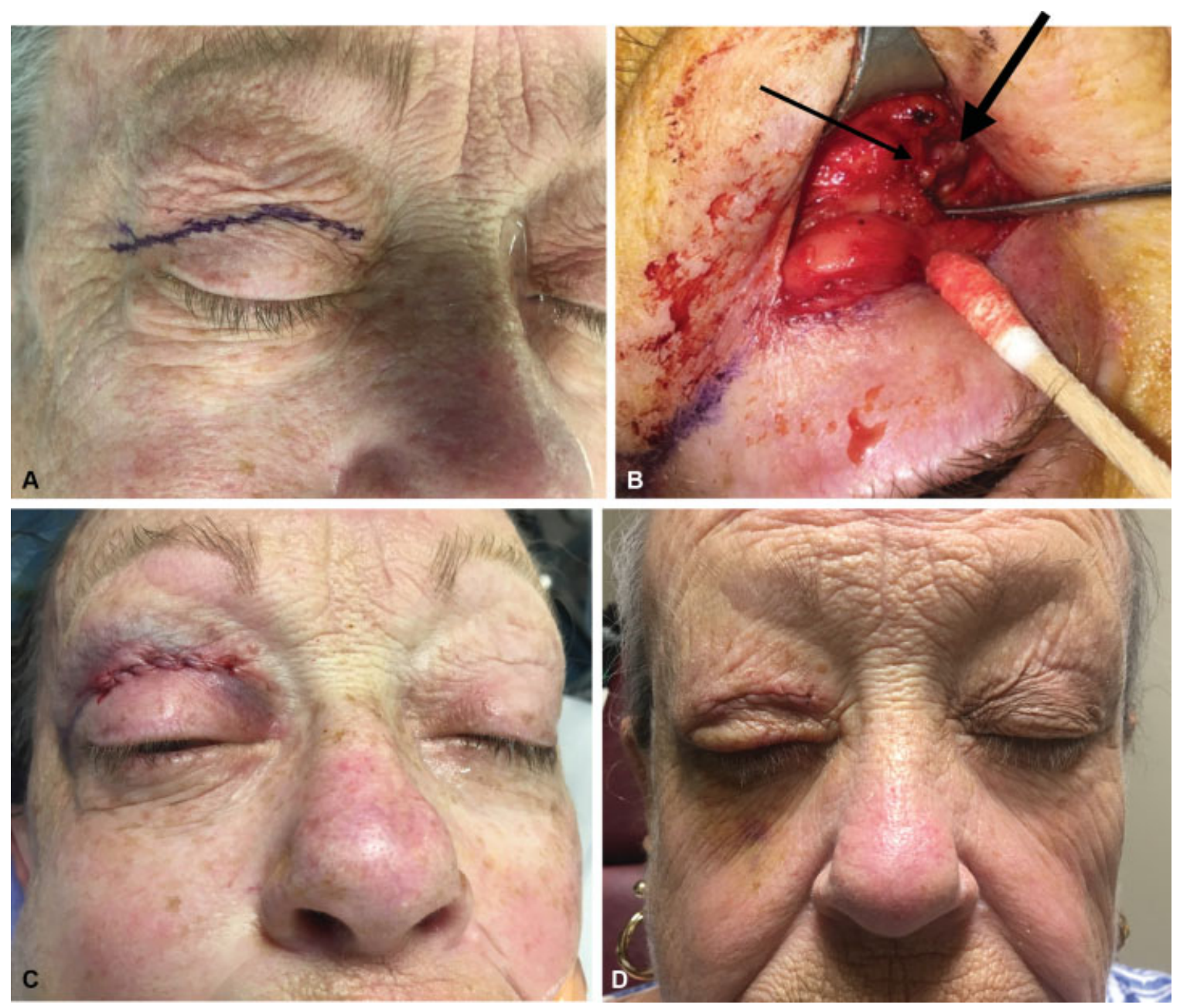

Fig. 2 (A) Preincisional marking. (B) Dissection to superior orbital rim, preserving the orbital septum. Wide arrow marks tumor. The supratrochlear nerve is immediately lateral to the mass (narrow arrow). (C) Cosmetic result upon closure. (D) Cosmetic result at 1-week postop.
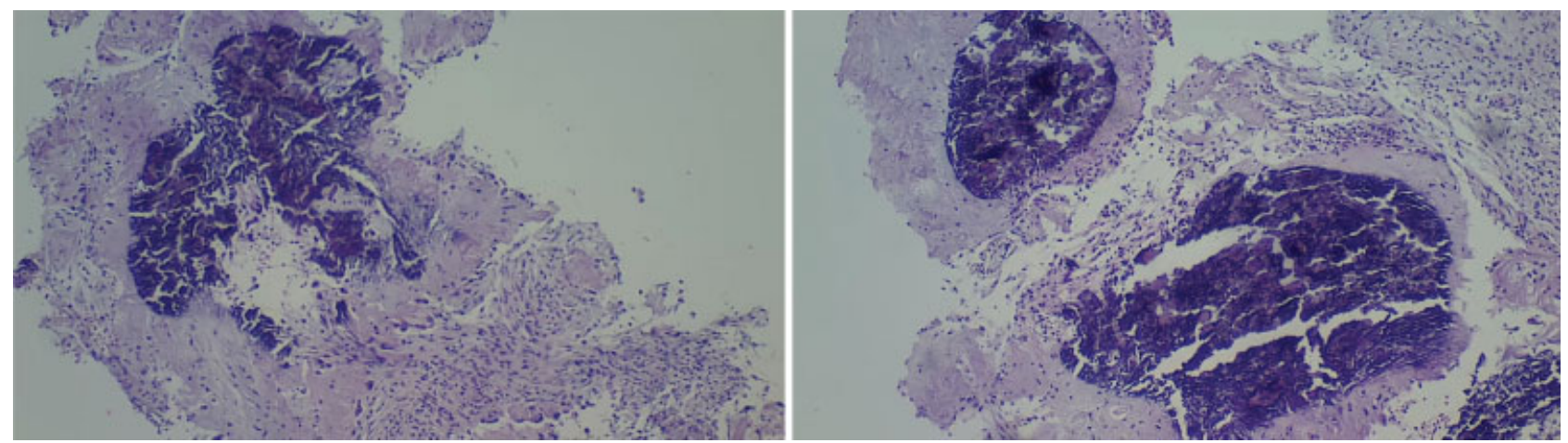

Fig. 3 The histological findings include central calcification with surrounding chondroid matrix organized in a nodular pattern. There is a bland spindle cell component with palisading around the nodules. An epithelial membrane antigen (EMA) immunohistochemical stain (not shown) is positive. Hematoxylin and eosin (H\&E) stain $100 \times$.

optimal access for open biopsy while affording the patient an aesthetically pleasing result. The postoperative course was uncomplicated and the ultimate cosmetic result was favorable (-Fig. 2D). Pathological analysis, while challenging, was consistent with the literature and made using the organized calcific structure of the mass and EMA immunohistostain positivity. The patient's decision to very closely watch the mass using serial imaging was made with the knowledge that if the mass were to expand, resection would be pursued knowing access to the area of interest is relatively straight forward. This is the first documented case of an anterior cranial fossa CAPNON involving the frontal sinus with a unique and minimally invasive approach to obtaining its pathologic diagnosis. 
e78 Anterior Cranial Fossa Calcifying Pseudoneoplasm of the Neuroaxis Blood et al.

\section{Conclusion}

We present the first known case of CAPNON involving the anterior skull base with erosion into the frontal sinus and superior orbital rim. The biopsy was performed using a minimally invasive transblepharoplasty approach resulting in the diagnosis of the mass and a cosmetically appealing result. While rare, we recommend that CAPNON remain on the differential for an aggressive appearing calcified mass of the anterior cranial fossa.

\section{Conflicts of Interest}

The views herein are the private views of the authors and do not reflect the official views of the Department of the Army or the Department of Defense. The authors have no funding, financial relationships, or conflicts of interest to disclose.

\section{References}

1 García Duque S, Medina Lopez D, Ortiz de Méndivil A, Diamantopoulos Fernández J. Calcifying pseudoneoplasms of the neuroaxis: report on four cases and review of the literature. Clin Neurol Neurosurg 2016;143:116-120
2 Rhodes RH, Davis RL. An unusual fibro-osseous component in intracranial lesions. Hum Pathol 1978;9(03):309-319

3 Mohapatra I, Manish R, Mahadevan A, Prasad C, Sampath S, Shankar SK. Calcifying pseudoneoplasm (fibro osseous lesion) of neuroaxis (CAPNON) - a case report. Clin Neuropathol 2010;29 (04):223-226

4 Bertoni F, Unni KK, Dahlin DC, Beabout JW, Onofrio BM. Calcifying pseudoneoplasms of the neural axis. J Neurosurg 1990;72(01): 42-48

5 Fletcher AM, Greenlee JJ, Chang KE, Smoker WR, Kirby PA, O'Brien EK. Endoscopic resection of calcifying pseudoneoplasm of the neuroaxis (CAPNON) of the anterior skull base with sinonasal extension. J Clin Neurosci 2012;19(07):1048-1049

6 Knipe TA, Gandhi PD, Fleming JC, Chandra RK. Transblepharoplasty approach to sequestered disease of the lateral frontal sinus with ophthalmologic manifestations. Am J Rhinol 2007;21(01):100-104

7 Chu EA, Quinones-Hinojosa A, Boahene KD. Trans-blepharoplasty orbitofrontal craniotomy for repair of lateral and posterior frontal sinus cerebrospinal fluid leak. Otolaryngol Head Neck Surg 2010; 142(06):906-908

8 Raza SM, Boahene KD, Quinones-Hinojosa A. The transpalpebral incision: its use in keyhole approaches to cranial base brain tumors. Expert Rev Neurother 2010;10(11):1629-1632

9 Owusu Boahene KD, Lim M, Chu E, Quinones-Hinojosa A. Transpalpebral orbitofrontal craniotomy: a minimally invasive approach to anterior cranial vault lesions. Skull Base 2010;20(04):237-244

10 Andaluz N, Romano A, Reddy LV, Zuccarello M. Eyelid approach to the anterior cranial base. J Neurosurg 2008;109(02):341-346 